\title{
Die Olympische Bewegung und das Geld
}

Holger Preuß

\section{Abstract}

Die Olympischen Spiele und das über sie erwirtschaftete Geld sind der treibende Motor für die Olympische Bewegung. Dieser Beitrag soll Aufklärung in die komplizierten Verflechtungen des olympischen Geldes bringen.

Die weltweite Organisation des Sports wird über das IOC mit der Bindung vieler Organisationen an seine Olympische Charta (der Satzung) gesteuert. Die Vermarktung der Olympischen Spiele schreitet ungebrochen voran und führt zu Einnahmen über $8 \mathrm{Mrd}$. USD pro Olympiade. Die Einnahmen durch die Spiele versorgen nicht nur die Organisationskomitees der Olympischen Spiele, 206 Nationale Olympische Komitees und 40 Internationale Sportfachverbände mit Geld, sondern auch zahlreiche andere Organisationen und den Breitensport. Letzterer wird vor allem über Olympic-Solidarity-Programme finanziell gefördert, in denen allein 590 Mio. USD verteilt werden.

Es zeigt sich, dass die Organisationskomitees der Spiele den größten Anteil des Geldes vom IOC bekommen und darüber hinaus noch eigene Einnahmen erwirtschaften. Damit stellt das IOC bisher sicher, dass die Organisationskosten der Spiele nur wenig von Steuergeldern finanziert werden. Allerdings sind oft infrastrukturelle Änderungen der an der Ausrichtung der Spiele beteiligten Städte notwendig. Diese wiederum sind von der Ausrichterstadt und damit dem Steuerzahler zu tragen, sollten dann aber auch über Jahrzehnte einen Nutzen bringen. Kostenüberschreitungen treten vor allem bei diesen langfristigen Investitionen auf.

\section{Die Finanzierung der Olympischen Bewegung}

Das Internationale Olympische Komitee (IOC) wurde am 23. Juni 1894 von Baron Pierre de Coubertin in Paris gegründet und hat seinen Sitz seit 1915 in Lausanne (IOC 2020a). Coubertin hat sein gesamtes Vermögen in den Aufbau der Olympischen Bewegung investiert. 


\section{Internationales Olympisches Komitee (IOC)}

Als Pierre de Coubertin 1892 in Paris die bevorstehende Wiedereinführung der Olympischen Spiele ankündigte, konnte sich niemand damals das Ausmaß des Projekts vorstellen, das die Schaffung einer internationalen Bewegung mit sich bringen würde. Das IOC wurde am 23. Juni1894 gegründet und ist die oberste Instanz der Olympischen Bewegung und Besitzer der Olympischen Spiele, die zuerst am 6. April 1896 in Athen durchgeführt wurden. Das IOC ist eine gemeinnützige, unabhängige internationale und von den Vereinigten Nationen anerkannte Non-Profit-Organisation, die sich dafür einsetzt, eine bessere Welt durch Sport zu schaffen. Als oberstes Organ der Olympischen Bewegung fungiert das IOC als Katalysator für die Zusammenarbeit zwischen den Nationalen Olympischen Komitees, den internationalen Sportfachverbänden, den Athlet*innen und den Organisationskomitees der Spiele bis hin zu den weltweiten olympischen Sponsoren, Medienpartnern. Das IOC sorgt mit einer Vielzahl von Programmen und Projekten neben den Olympischen Spielen für den Erfolg und die Verbreitung der olympischen Werte.

Heute ist das IOC eine internationale Nichtregierungsorganisation in der Rechtsform eines Vereins nach Schweizer Recht mit dem explizit pro-sozialen Bestreben, Bildung und Frieden zu fördern (IOC 2020a). Damit sichert das IOC seine moralische Legitimität. Mit Hilfe der starken Kommerzialisierung fördert es den Olympismus, der in der Olympischen Charta in sieben Prinzipien festgeschrieben ist. Prinzip 3 definiert die Idee und das Eigentum und damit die kognitive Legitimität der Olympischen Bewegung und Olympischen Spiele:

„The Olympic Movement is the concerted, organised, universal and permanent action, carried out under the supreme authority of the IOC, of all individuals and entities who are inspired by the values of Olympism. It covers the five continents. It reaches its peak with the bringing together of the world's athletes at the great sports festival, the Olympic Games" (IOC 2020a, S. 11).

Von 2013 bis 2016 erwirtschaftete das IOC mit den Olympischen Spielen ca. 5,7 Mrd. USD. Davon bleiben ca. $10 \%$ beim IOC als Organisation und $90 \%$ werden an vom IOC anerkannte Organisationen der Olympischen Bewegung weitergegeben. Die Spiele sind somit eine wesentliche Finanzierungsquelle der Olympischen Bewegung. Dieses Geld sichert dem IOC aber auch seine praktische Legitimität und damit seine Macht in der Führung des Weltsports. 
Die Olympische Bewegung umfasst den Großteil der Organisationen im Weltsport und besteht im Kern aus drei Bereichen:

1. dem IOC,

2. 40 Internationalen Sportverbänden (IF) und

3. 206 Nationalen Olympischen Komitees (NOC).

Die NOCs und IFs sind Dachverbände und daher sind auch alle ihre Mitglieder, also die nationalen Verbände, Sportvereine und Personen (Athlet"innen, Kampfrichter*innen, etc.) Teil der Olympischen Bewegung. Außerdem zählen die Organisationskomitees für die Spiele dazu:

4. Olympischen Sommer und Winter Spiele (OCOGs) und

5. Youth Olympic Games (YOG) im Sommer und Winter.

Schließlich gehören zur Olympischen Bewegung noch einige vom IOC anerkannte Organisationen und Verbände, z.B.:

6. das Internationale Paralympische Komitee oder

7. IFs, deren Sportarten ggf. bald ins Olympische Programm aufgenommen werden. Dazu müssen diese aber vom IOC anerkannt sein.

\section{Der Aufbau des IOC und die Zuständigkeiten aus finanzieller Sicht}

Das IOC ist ein Verein nach Schweizer Recht ( $\$ 60 \mathrm{ff}$ ) des Schweizerischen Zivilgesetzbuches. Dies gibt die interne Struktur des IOC und die Rolle seiner Organe (IOC-Session, IOC-Exekutive, Präsident) vor. Der IOC Exekutive kommt eine Schlüsselrolle zu, da sie die allgemeine Gesamtverantwortung für die Verwaltung des IOC und die (finanzielle) Führung seiner Geschäfte übernimmt. Sie genehmigt alle internen Regelungen, die sich auf das IOC beziehen, sie erstellt einen Jahresbericht und die Finanzberichte. Außerdem nimmt sie alle Aufgaben wahr, die nicht durch das Gesetz oder die Olympische Charta, die IOC-Session (jährliche IOCVollversammlung) oder den Präsidenten zugewiesen sind. Insgesamt hat die IOC-Exekutive weitreichende Befugnisse, die sie aber auch an seine Mitglieder, die IOC-Kommissionen, an die IOC-Verwaltung oder an Dritte delegieren kann. Die IOC-Verwaltung unter der Leitung des IOC-Generaldirektors erstattet der IOC-Exekutive regelmäßig Bericht.

Die Verwaltung des IOC untersteht dem Generaldirektor (DG), der das IOC mit Unterstützung der Direktoren leitet. Neben den Büros für den Präsidenten und den DG ist die Verwaltung in Abteilungen unterteilt, die je nach Umfang unterschiedlich groß sind und von Direktoren geleitet 
werden. Jede Abteilung ist in Referate unterteilt (die größten mit mehr als 40 Personen), die für die geschäftlichen Aspekte in ihrem jeweiligen Zuständigkeitsbereich verantwortlich sind.

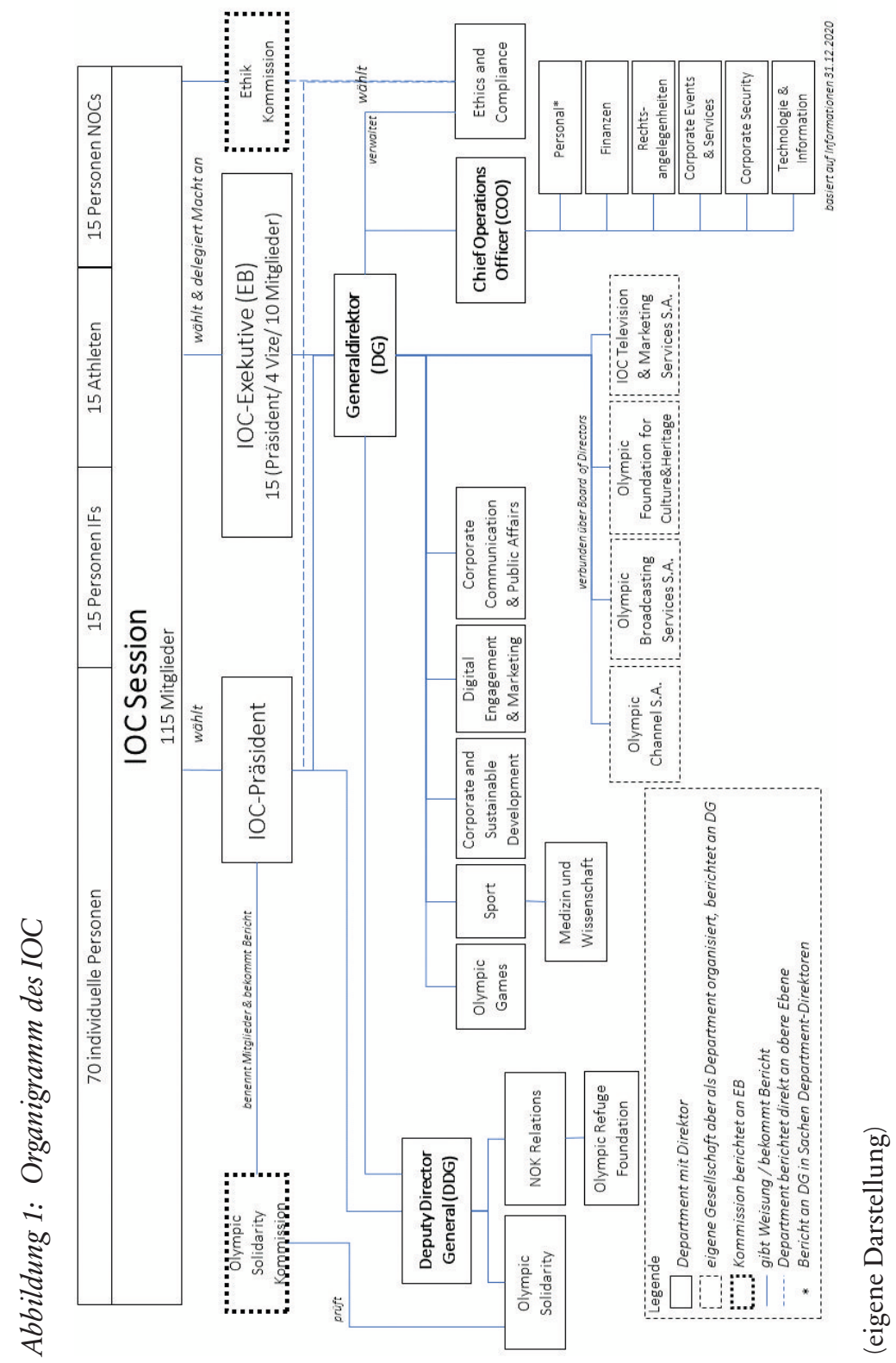


Abb. 1 zeigt, dass die Aufgabenteilung des IOC nicht nur durch direkt unterstellte Abteilungen strukturiert ist, sondern auch weitgehend autonome Organisationen betreibt. Insbesondere tut das IOC dies durch private Gesellschaften mit beschränkter Haftung (S.A. ist die französische Abkürzung) in den Bereichen Marketing, Medien und Fernsehen, wobei die Olympic Foundation (Stiftung) jeweils alle Anteile dieser Gesellschaften hält. Der IOC-Präsident ist Vorsitzender des Stiftungsrates, der das Leitungsorgan der Olympic Foundation ist. Das IOC hat mehrere verschiedene juristische Personen geschaffen, um bestimmte Aufgaben zu erfüllen (Abb. 2). Die Struktur erscheint kompliziert, da rechtlich unabhängige Organisationen (Stiftungen) und Gesellschaften (S.A.) in die IOC-Struktur integriert sind. Sie schließen jedoch rechtlich eigene Verträge $\mathrm{ab}$ und können steuerliche Rechnungen ausstellen. In Abb. 1 sind diese gestrichelt dargestellt, da sie dem DG unterstellt sind, der in ihrem Vorstand mitarbeitet.

Abbildung 2: Organisation der IOC-Gruppe im Jahr 2020

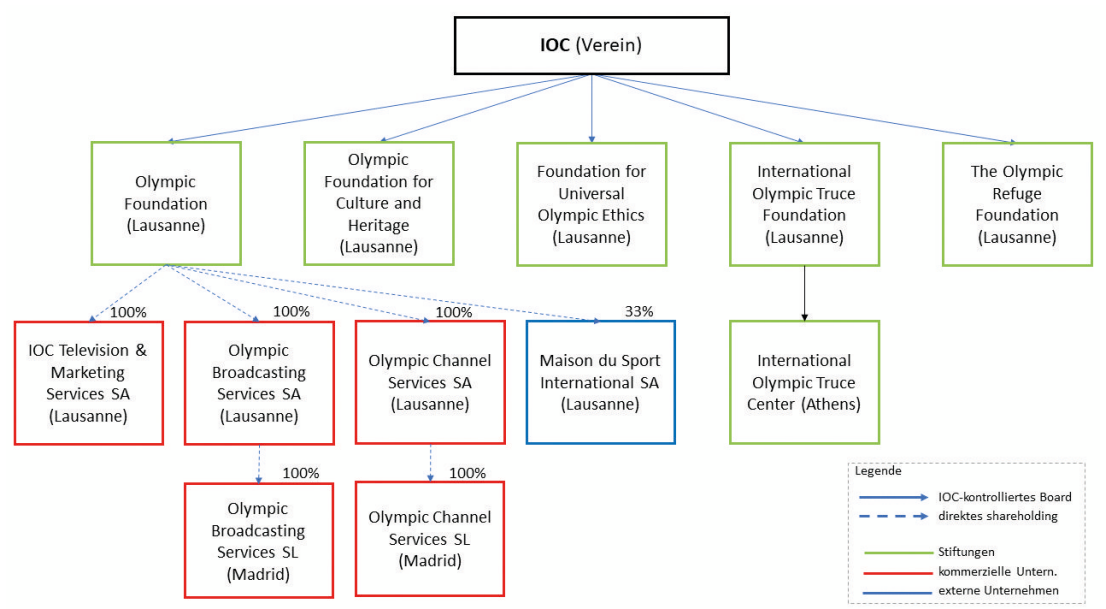

(eigene Darstellung)

Einige Organisationen der IOC-Gruppe sind Stiftungen nach Schweizer Recht. Dies ist insbesondere der Fall bei der Olympische Stiftung für Kultur und Erbe, die Olympic Foundation und Olympische Stiftung für Flüchtlinge. Sie sind in unterschiedlichem Maße im Vorstand des IOC vertreten und regeln ihre Aktivitäten mit einer gewissen operativen Autonomie vom IOC. Gemäß Artikel 80ff. des Schweizerischen Zivilgesetzbuches 
sind Stiftungen eine Gruppe von Vermögenswerten, die von einem Stifter einem bestimmten ideellen Zweck zugewiesen wurden und die unter die Verwaltung eines Stiftungsrates gestellt werden, der unter der Aufsicht staatlicher Behörden arbeitet und überwacht, dass die Ausgaben in Übereinstimmung mit dem satzungsgemäßen Auftrag der Stiftung und den geltenden Gesetzen erfolgen. Das IOC ist der Stifter der oben genannten Stiftungen und durch die Stiftungssatzung hat das IOC als Stifter bestimmte Vorrechte, die ein gewisses Maß an faktischer Kontrolle ermöglichen (das Maß an Kontrolle ist nicht für alle vom IOC gegründeten Stiftungen gleich), insbesondere im Hinblick auf die Regeln zur Ernennung der Mitglieder der Stiftungsräte. Der IOC-Präsident verwaltet die Stiftungen nicht, aber er ist der Vorsitzende der jeweiligen Stiftungsräte.

Andere Einrichtungen wurden vom IOC als private Gesellschaften mit beschränkter Haftung gegründet, um bestimmte Geschäftsaufgaben zu erfüllen. Dies ist der Fall bei IOC Television Marketing \& Services S.A. (Schweiz), Olympic Broadcasting Services S.A. (Schweiz) und ihrer hundertprozentigen Tochtergesellschaft Olympic Broadcasting Services S.L. (Spanien), Olympic Channel Services S.A. (Schweiz) und ihrer hundertprozentigen Tochtergesellschaft Olympic Channel Services S.L. (Spanien). Dabei handelt es sich um private Gesellschaften, die sich im Besitz der Olympischen Stiftung befinden. Sie sind als solche keine IOCAbteilungen (daher in Abb. 1 mit gestrichelten Linien), da sie eigenständige juristische Personen sind, wohl aber in unterschiedlichem Maße in die Operationen des IOC integriert. Ihre Vorstände bestehen aus IOCDirektoren, IOC-Mitgliedern und organisationsfremden Personen. Sie alle erbringen ihre Leistungen fast ausschließlich für das IOC, das im Gegenzug ihre operativen Kosten übernimmt. Bei diesen Unternehmen handelt es sich nicht um eigenständige Geschäftsbetriebe oder gewinnorientierte Wirtschaftsunternehmen. Das IOC hat Dienstleistungsunternehmen gegründet, um bestimmte Aufgaben professionell zu bewältigen.

Das IOC schüttet ca. 90 Prozent seiner Einnahmen wieder aus. In ihren Rollen als gemeinnützige Organisation profitiert das IOC sowie die Olympische Stiftung für Kultur und Erbe, die Olympic Foundation und die Internationale Stiftung für den Olympischen Frieden von einer vollständigen Befreiung der direkten Steuer in der Schweiz gemäß der Vereinbarung zwischen dem IOC und dem Schweizer Bundesrat vom 1. November 2000 (IOC 2019, S. 50). Die Gesellschaften (S.A.) zahlen allerdings eine Abgabe auf ihre Betriebskosten sowie das IOC insgesamt spezifische Steuern (z.B. Importsteuern, Grundsteuer etc.) (IOC 2019, S. 50). 
Um die Kompetenzbereiche professionell und von den IOC-Mitgliedern geführt zu halten, existieren insgesamt 31 Kommissionen, von denen 27 durch externe Experten erweitert werden. Die Experten sind Personen mit hohem Fachwissen und außerdem meist Vertreter*innen von Organisationen, deren Wissen und Netzwerke das IOC mit einbeziehen will. IOCKommissionen erfüllen einen wichtigen Zweck. Sie spiegeln die Bereiche wider, die für das IOC und die Olympischen Spiele wichtig sind. Die Abteilungen helfen bei der Vorbereitung der Arbeit der IOC-Kommissionen, berichten über ihre Aktivitäten an die jeweilige Kommission, die die Abteilungen wiederum anleitet. In diesem Sinne arbeiten Kommission und Abteilung komplementär und profitieren voneinander, mit dem Unterschied, dass die Kommissionen von IOC-Mitgliedern und nicht von Verwaltungsangestellten geleitet werden. Die Kommissionen haben keine Entscheidungsbefugnis (IOC 2020a, $\mathbb{2 1}$ ), sondern dienen der Beratung der IOC-Exekutive und des Präsidenten und letztlich der IOC-Session (IOC 2019, 45). Die Tatsache, dass den meisten Kommissionen mehrere IOC-Mitglieder angehören, bedeutet, dass die IOC-Session zu jedem Thema von fachlich informierten Personen begleitet wird, die sich in Diskussionen zu Wort melden können.

Für die (finanzielle) Transparenz ist das Audit Committee besonders wichtig. Es soll die Integrität der Jahresabschlüsse des IOC überwachen und stellt sicher, dass die Jahresabschlüsse des IOC und seiner Tochtergesellschaften (Abb. 2) in Übereinstimmung mit den International Financial Reporting Standards (IFRS) erstellt werden und dem Schweizer Recht sowie der Olympischen Charta entsprechen (IOC 2019, S. 46). Es soll wichtige Beschlüsse zur Finanzberichterstattung überprüfen und letztlich eine Risikobeurteilung und Bewertung der internen Kontroll- und Risikomanagementsysteme des IOC an die Exekutive sicherstellen. Eine Verbindung zur Ethikkommission besteht, indem Bedenken über mögliche Unregelmäßigkeiten in ethischen Fragen oder der Finanzberichterstattung vorgebracht werden sollen. Diese Kommission empfiehlt den Einsatz eines externen Prüfers (IOC 2019, S. 47). Deren Empfehlung wird von der IOCExekutive geprüft und schließlich über die IOC-Session gewählt und besetzt. Gegenwärtig (Stand 2021) ist dies ein Team von PricewaterhouseCoopers (PwC). Die externe Revisionsstelle gibt ein Urteil darüber ab, ob die konsolidierte Jahresrechnung den IFRS und den anwendbaren Gesetzen und Standards entspricht.

Der IOC-Präsident gründet Kommissionen, wann immer es notwendig erscheint (IOC 2020a, $\mathbb{\$} 21$ ). Somit kann das IOC direkt und schnell auf Umweltveränderungen reagieren. Eine wichtige Umweltänderung ist die Digitalisierung, die 2019 die Gründung der Abteilung Digital Engagement 
and Marketing nach sich zog und von der Digital and Technology Commission seither unterstützt wird. Eine weitere neue Abteilung ist die für Corporate and Sustainable Development. Sie wird von der Sustainability and Legacy Commission unterstützt. Bei beiden ist das wichtige Thema der Legacy (Olympisches Erbe) verankert.

\section{Die Finanzierung der Olympischen Bewegung durch das IOC}

Innerhalb des IOC liegt die oberste Macht bei der IOC-Session. Die Befugnisse der Session sind in der Olympischen Charta definiert (IOC 2020a, $\$ 18$ ), die unter anderem die Verantwortung für die Genehmigung des Jahresberichts und des Jahresabschlusses des IOC sowie für die Ernennung des unabhängigen Rechnungsprüfers des IOC beinhalten. Auf administrativer Ebene wird das Budget von einem Plan- und Budgetkomitee (bestehend aus bestimmten IOC-Direktoren) geplant und kontrolliert und vom Generaldirektor genehmigt und schließlich von der Exekutive bewilligt. Dieses Plan- und Budgetkomitee überwacht und kontrolliert den 4-JahresBudgetplan (pro Olympiade) und prüft und genehmigt individuell die Budgets, die den Aktivitäten und Projekten der Abteilungen zugewiesen werden, die die Mittel entsprechend ihrer ständigen Aufgaben und Projekte ausgeben. Das Plan- und Budgetkomitee prüft die Projektanträge im Hinblick auf ihre Abteilungszugehörigkeit, um sicherzustellen, dass Synergien genutzt, Ausgaben minimiert und Beschaffungs- und Rechtsverfahren eingehalten werden.

Alle vom IOC anerkannten Organisationen (Abb. 3) verpflichten sich im Gegenzug für das Geld und die anderen Vorteile, die sie erhalten, den in der Satzung des IOCs (Olympische Charta) für ihre Organisation festgeschrieben Auflagen zu folgen. Das heißt, dass alle diese Organisationen ihre Statuten und Aktivitäten in Einklang mit der Olympischen Charta bringen müssen, um anerkannt zu bleiben. Allerdings behält jeder internationale Weltverband seine Autonomie in der Führung ihres Sports (IOC 2020a, $\$ 25)$. 
Abbildung 3: Organisationen der Olympischen Bewegung finanziert durch Olympische Spiele

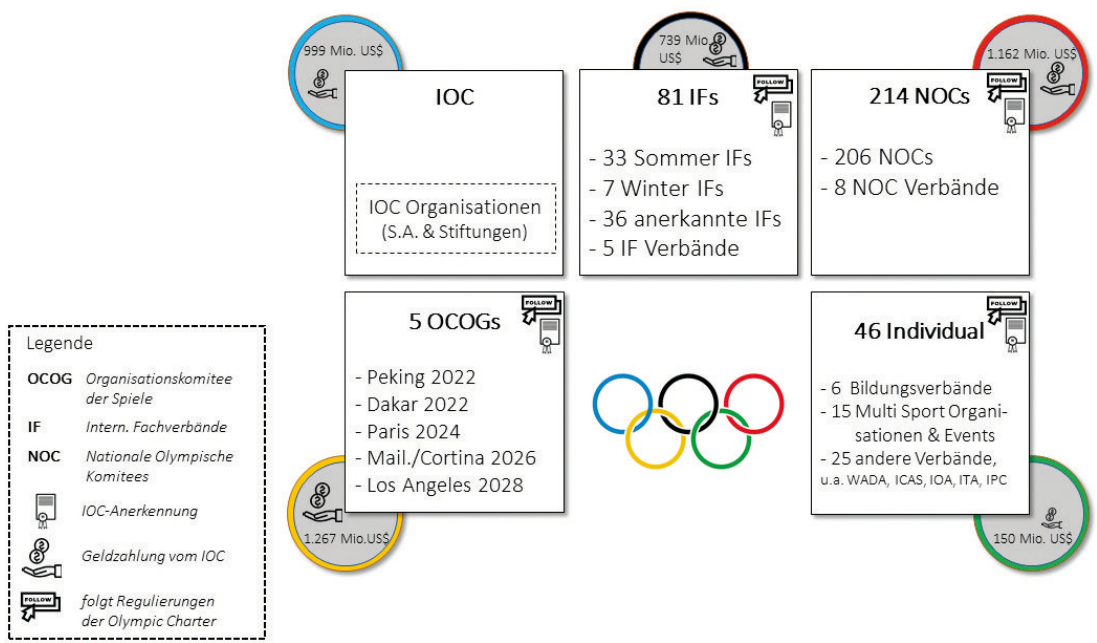

(IOC, 2019, 120f.; IOC, 2020b; IOC, 2020a, \$45.1.3)

Bei den NOCs ist die Regelung strikter, denn sie werden vom IOC nur dann anerkannt, wenn sie die Einhaltung der Olympischen Charta in ihrem Land gewährleisten. Nur dann können sie Mannschaften zu den Olympischen Spielen und den YOG senden, sich um die Ausrichtung der Spiele bewerben oder finanzielle Unterstützung bekommen. So ist das IOC über die NOCs territorial auf der ganzen Welt vertreten und verbreitet seine Grundideen, die sogenannten „Fundamental Principles“ (IOC 2020a, $\$ 27.2 .2$ ). Eine wichtige regulative IOC-Vorgabe ist, dass die Mehrheit der NOC-Mitgliederstimmen von den Nationalen Olympischen Sportverbänden kommen muss (IOC 2020a, \$28.3). Außerdem dürfen Regierungen keine Funktionäre eines NOC bestimmen, wobei die Mitglieder eines NOC durchaus Regierungsvertreter ins Amt wählen dürfen (IOC 2020a, $\mathbb{S} 28.4$ ), wie dies z.B. in China und Belarus gegenwärtig der Fall ist.

Alle IOC-anerkannten Organisationen erhalten eine finanzielle Unterstützung vom IOC (Abb. 4). Alle NOCs können von weiteren OlympicSolidarity-Förderungen profitieren, die im Zeitraum von 2021 bis 2024 ca. 590 Mio. USD betragen (IOC 2020d). Diese Summe ist im Schaubild unter NOC enthalten. Es fällt auf, dass IFs und NOCs unterschiedlich viel Geld 


\section{Holger Preuß}

bekommen und die Organisationskomitees der Spiele (OCOGs) mit über $40 \%$ den größten Anteil.

Abbildung 4: Einnahmen und Ausgaben des IOC über 4 Jahre in Prozent

\section{IOC Ausgaben}

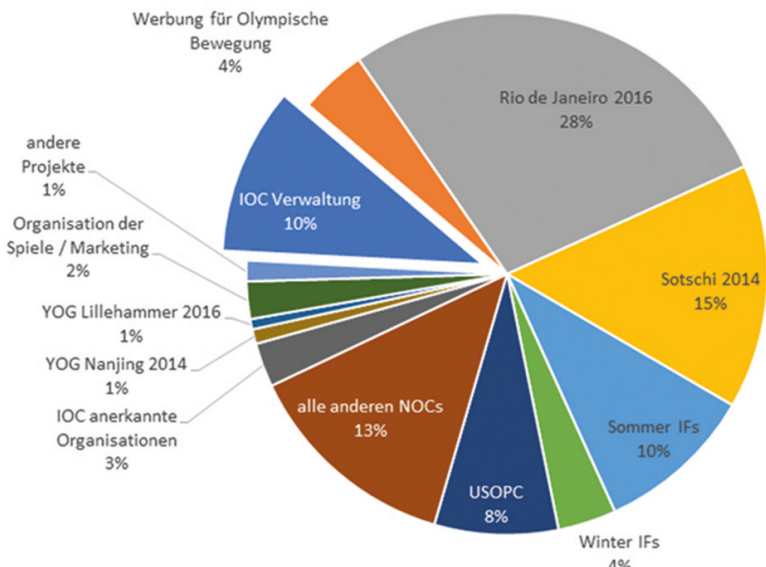

IOC Einnahmen

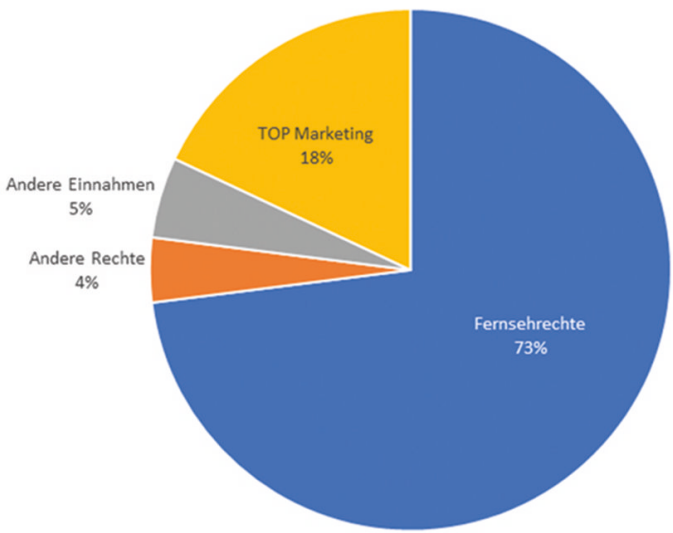

(IOC, 2019) 
Die folgende Abbildung gibt einen groben Überblick über die Einnahmen und Ausgaben des IOC über eine Olympiade, den Zeitraum von vier Jahren zwischen den Olympischen Sommerspielen. Belastbare Daten lagen nur für die Olympiade 2013-2016 vor. Die Aussage in Prozenten bringt aber gut zum Ausdruck, wie die Einnahmen des IOC in etwa verteilt werden.

Die hier aufgeführten Einnahmen sind nur der Teil der gesamten Einnahmen durch die Olympischen Spiele, die das IOC eingenommen hat. Die Organisationskomitees der Spiele erhalten weitere Einnahmen z.B. durch den Verkauf von Eintrittskarten, Merchandising, nationalen Sponsoring-Rechten, die überwiegend im OCOG verbleiben und nur wenige Prozent davon werden an das IOC abgegeben. Die Einnahmen des IOC ergeben sich größtenteils durch die Vermarktung der TV-Rechte sowie durch das seit 1985 aufgesetzte internationale Marketingprogramm (TOP). Andere Rechte beinhalten Einnahmen aus der kommerziellen Verwertung des olympischen Symbols (Ringe) und der olympischen Embleme (z.B. Logos der Spiele). Die anderen Einnabmen setzen sich aus weiteren Medienrechten (unilaterale und paralympische Übertragungen) zusammen.

Von der Olympiade Athen (2001-2004) bis Rio de Janeiro (2013-2016) konnte das IOC seine Einnahmen von 3 auf 5,7 Mrd. USD nahezu verdoppeln und für die Olympiade 2017-2020 ist eine weitere Steigerung absehbar.

Auffallend ist, dass das amerikanischen NOC (USOPC) gut $36 \%$ der Mittel bekommt, die alle anderen 205 NOCs erhalten. Dies liegt an einem Vertrag, der dem USOPC $20 \%$ der Einnahmen aus dem TOP-Programm zusagt. Da diese gegenwärtig (2017-2020) extrem gestiegen sind, dürfte das USOPC für die kommenden Jahre finanziell noch sehr viel besser ausgestattet sein, als in der Abbildung dargestellt ist (Owen, 2019).

Es macht finanziell einen großen Unterschied, ob eine Organisation lediglich vom IOC anerkannt ist oder ob ihre Sportart Teil des Olympischen Programms ist. Um den Einfluss ihrer jeweiligen Gruppe (IFs, NOCs etc.) bei Verhandlungen gegenüber dem IOC zu stärken, haben sich Dachverbände gebildet, z.B. Association für NOCs (ANOC) für alle NOCs der Welt, Association für Olympische Sommer IFs (ASOIF), Association für Olympische Winter IFs (AIOWF), Association für IOC-anerkannte IFs (ARISF). Über $40 \%$ der finanziellen Unterstützung des IOC geht allerdings an die OCOGs. Der Anteil an die IFs berechnet sich über viele Kriterien, letztlich aber über die Attraktivität der Sportart für die Medien und damit für die Olympischen Spiele. So haben z.B. Segeln, Kanu, Fechten, Handball und Ringen (4. Gruppe) ca. 12 Mio. USD für Rio 2016 erhalten 
und Basketball, Fußball, Radsport, Volleyball und Tennis (2. Gruppe) ca. 26 Mio. USD. In der obersten Gruppe ist die Leichtathletik mit ca. 45 Mio. USD vertreten. Für Tokio 2020 sind ca. dieselben Summen vorgesehen. Dies zeigt eingehend, wie wichtig die Olympischen Spiele aus monetärer Sicht für die Internationalen Verbände sind.

Die Beteiligung der NOCs an den Einnahmen setzt sich erstens aus einem Grundbeitrag zur Sicherung der Unabhängigkeit des NOCs zusammen, zweitens aus Zuwendungen über Olympic-SolidarityProgramme sowie drittens aus der Wirtschaftskraft des Landes für die Sponsoren. Letzterer Punkt begründet sich damit, dass die Nationen ihr Gebiet für die internationalen Sponsoren exklusiv freihalten und dafür am TOP-Programm beteiligt werden.

Im Gegenzug für die finanziellen Zuwendungen verpflichten sich alle IOC-anerkannten Verbände die für sie festgeschrieben Vorgaben der Olympischen Charta umzusetzen, d.h. sie folgen z.B. dem WeltAnti-Doping-Code, schaffen grundlegende Good-Governance-Strukturen, bekämpfen die Manipulation des Spielbetriebs und halten sich politisch unabhängig von der nationalen Regierung. Wird gegen die Olympische Charta verstoßen, so kann das IOC Sanktionen aussprechen. Diese reichen vom Entzug der Geldzuweisungen, über die Limitierung von zu entsendenden Athlet"innen zu den Olympischen Spielen (Beispiel Gewichtheben 2016), bis zum Ausschluss von den Spielen (Beispiel Boxverband AIBA 2020) oder gar dem Ausschluss der gesamten Sportart (Beispiel Ringen 2013). Auch NOCs (oder ihre Funktionäre) können von den Spielen bzw. in einzelnen Jahren ausgeschlossen werden (Beispiel Indien 2013; Kuwait 2016; Russland 2018; Weißrussland 2021). Das IOC unterstützt seine IOCanerkannten Organisationen auch inhaltlich, indem es bei der Verbreitung der Olympischen Ideale, dem Kampf gegen Manipulation, Doping, Rassismus etc. hilft sowie Projekte zu Umweltschutz, Breitensport und der Gesundheit von Athlet*innen unterstützt. Außerdem ermöglicht eine IOCAnerkennung auch die Teilnahme an den Olympischen Spielen, Olympischen Jugendspielen und allen damit verbundenen Veranstaltungen (IOCSession, Olympic Forum, Olympische Kongresse). Die Olympischen Spiele sorgen für mediale Sichtbarkeit, verleihen Staaten Soft Power, schaffen nationalen Stolz und sportliche Vorbilder.

Die Kommission für Olympic Solidarity hat gemäß Regel 5 der Olympischen Charta einen besonderen Auftrag und die Verantwortung über die folgenden Programme zur Unterstützung des Weltsports. Das Budget, das von 509 Mio. USD (2017-2020) auf 590 Mio. USD (2021-2024) gesteigert wurde, verteilt sich bisher zu $41 \%$ auf Weltprogramme (für NOCs zur Entwicklung von Sport), zu $44 \%$ auf Kontinentalprogramme (Projekte 
von NOCs nach Kontinent) und zu $10 \%$ auf NOCs zur Sicherung der Teilnahme an den Olympischen Spielen. Die fehlenden $5 \%$ werden für Verwaltung und technische Unterstützung verwendet. Diese Unterstützung soll den NOCs helfen, sich zu professionalisieren, effiziente Strukturen zu schaffen und Training auf verschiedenen Leistungsebenen zu organisieren (IOC 2019, S. $22 \& 80-81$ ).

Die Verteilung der 2013-2016 erwirtschafteten 5,7 Milliarden USD ist sehr komplex. Die Ressourcenverteilung des IOC an seine anerkannten Organisationen ist in seinen Grundzügen in Abb. 3 dargestellt. Die Verteilung von Ressourcen an die IOC-anerkannten Organisationen des Weltsports läuft nach einem Verteilungsschlüssel. Vorrangiges Ziel der Ressourcenverteilung ist, dass die Organisationen politisch unabhängig bleiben können.

Zwei im Weltsport sehr wichtige Organisationen sind über Ressourcen bzw. Personen ebenfalls eng an das IOC gebunden. Die WADA (World Anti-Doping Agency) und das ICAS (International Council Of Arbitration for Sport). Um den Sportgerichtshof CAS (Court of Arbitration) autonomer zu machen, wurde der ICAS, eine Stiftung, als oberste Institution des CAS gegründet. ICAS führt und finanziert den CAS. Das ICAS erhält vom IOC 2019 eine finanzielle Unterstützung von ca. 7.5 Mio. USD (IOC 2019, S. 163), weit mehr bekommt allerdings die WADA, die bis heute zu $50 \%$ durch das IOC finanziert wird, ohne eine direkte Gegenleistung zu erbringen. Für 2021 sind dies 20,2 Mio. USD (WADA 2021). Obendrein ist das IOC informell in beiden Institutionen involviert, da es eine gewisse Anzahl von Mitgliedern im Vorstand stellt. Im Detail erfolgt dies durch Personalunion als IOC-Mitglied und Board Member im ICAS (4 von 20 ) oder der WADA (11 von 36). Dadurch erhält das IOC zum einen Informationen und kann zum anderen auch Informationen und Stimmgewicht zu Entscheidungen einbringen.

\section{Die Finanzierung der Olympischen Spiele}

Die IOC-Ausgaben (Abb. 4) gehen zu ca. $40 \%$ an die Ausrichterstädte und bilden für diese die sogenannte IOC-Contribution. Diese steigen von Spielen zu Spielen und sind in Abb. 5 dargestellt. 
Abbildung 5: IOC-Contribution (in Mio. USD) für die Ausrichter der Spiele 2002-2028

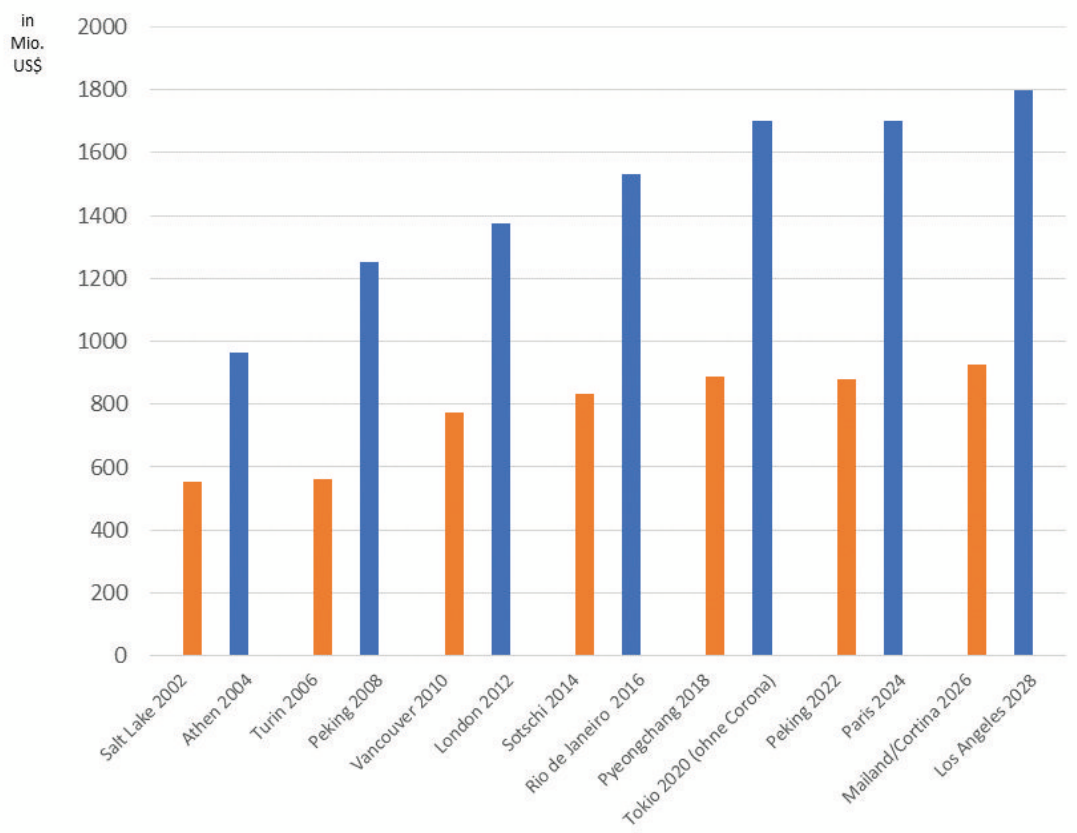

(IOC, 2020f; Host City Contracts 2022, 2024, 2026, 2028)

Die OCOGs haben weitere Einnahmen (nationale Sponsorenprogramme, Eintrittskarten, Merchandising etc.), von denen das IOC laut Host-CityContract eine Lizenzabgabe von 1-7,5\% (IOC 2017, \$24.8) erhält. In Abb. 6 und 7 sind die Einnahmen und Ausgaben der OCOGs für Sommer- und Winterspiele dargestellt. 
Abbildung 6: Einnahmen der OCOGs Rio de Janeiro 2016 und Pyeongchang 2018

Rio de Janeiro 2016

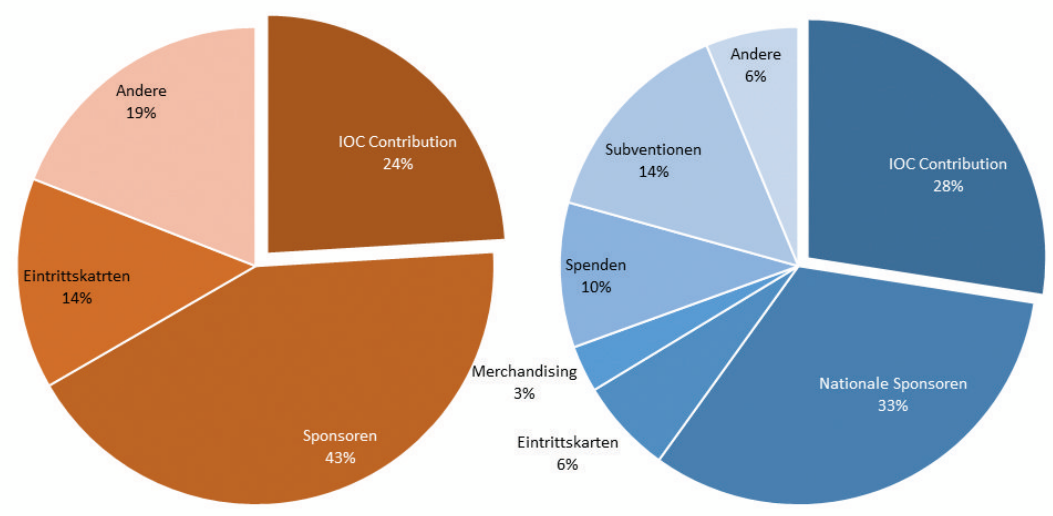

(Preuss et al., 2018)

Abbildung 7: Ausgaben der OCOGs Rio de Janeiro 2016 und Pyeongchang 2018

Rio de Janeiro 2016

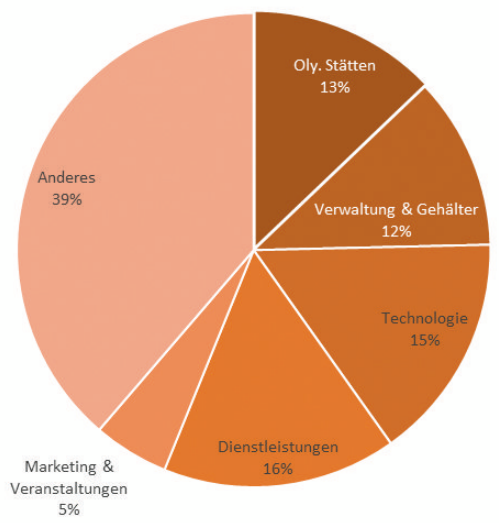

PyeongChang 2018

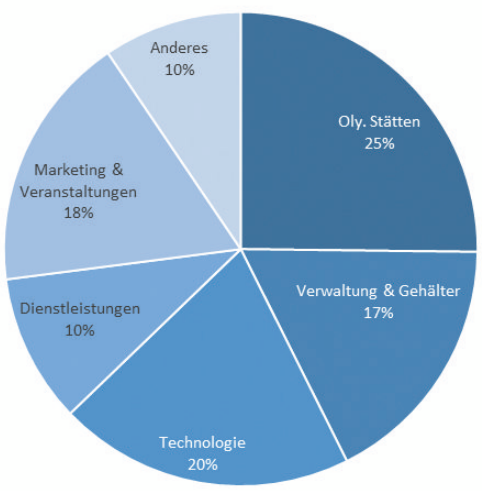

(Preuss et al., 2018) 
Die drei größten Einnahmequellen eines OCOGs sind die von den nationalen Sponsoren (Official Partner), der Verkauf von Eintrittskarten sowie der Verkauf von Lizenzen für Merchandising-Artikel (Tab. 1):

Tabelle 1: Daten zur Vermarktung Olympischer Spiele

\begin{tabular}{|c|c|c|}
\hline & $\begin{array}{l}\text { Rio de Janeiro } \\
2016\end{array}$ & $\begin{array}{l}\text { Pyeongchang } \\
2018\end{array}$ \\
\hline Sponsoren „Worldwide Partners“ & 11 & 13 \\
\hline Sponsoren „Official Partner“ & 7 & 11 \\
\hline Official Sponsor & 12 & 13 \\
\hline Official Supplier & 30 & 26 \\
\hline Anzahl Eintrittskarten & 6,2 Mio. & 1,1 Mio. \\
\hline Anzahl Merchandising-Produkte & 5.000 & 1.500 \\
\hline Lizenznehmer (für Merchandising) & 59 & 4 \\
\hline
\end{tabular}

(IOC, 2016; IOC, 2018; IOC, 2020f)

Abb. 8 zeigt die Entwicklung der einzelnen Finanzierungsquellen über die letzten fünf Olympiaden. Die Schwankungen in den Einnnahmen sind u.a. durch die wirtschaftliche Stärke des jeweiligen Gastgebers der Spiele bestimmt. Beispielsweise konnten Eintrittskarten in Großbrittanien 2012 teurer verkauft werden als in Griechenland 2004, da das OCOG und IOC generell bei der Preisfestsetzung auf die Kaufkraft der einheimischen Bevölkerung achten. Insgesamt zeigt sich aber ein beeindruckender Anstieg der Einnahmen. Das erreichte Plateau könnte mit dem wirtschaftlich schwächeren Rio 2016 gegenüber Vancouver 2010 und London 2012 zu tun gehabt haben, allerdings auch damit, dass ein gewisses kommerzielles Ausmaß der Spiele erreicht wurde. Für die Olympiade von Tokio 2020 (2017-2021) wird allerdings ein weiterer Anstieg erwartet. 
Abbildung 8: Einnahmequellen durch Olympische Spiele in Mio. USD

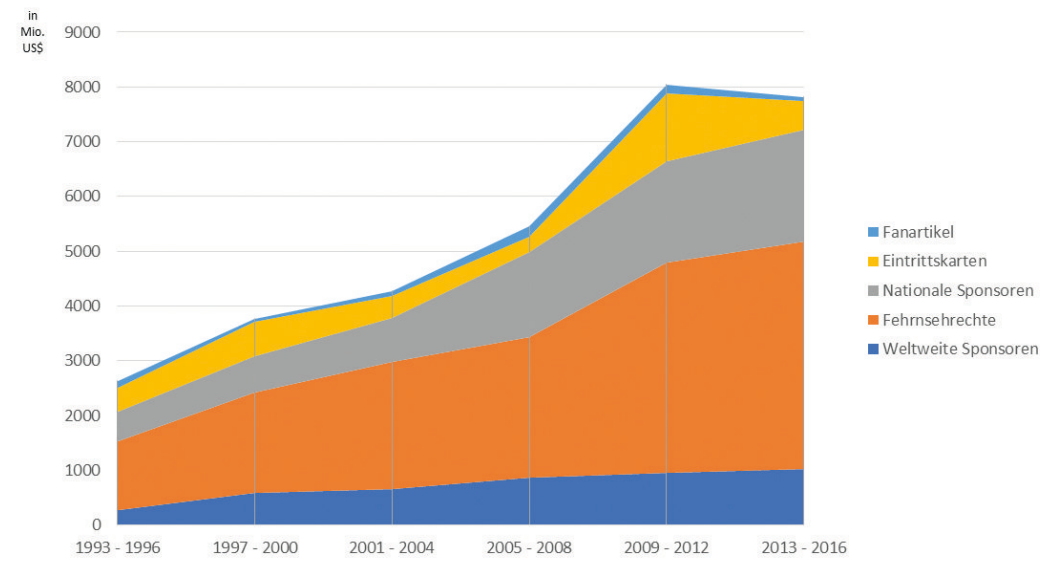

(IOC, 2020f)

Viele Unternehmen sehen in den Spielen eine einzigartige Gelegenheit, Geschäfte anzubahnen, Kontakte zu knüpfen und ihr Image zu verbessern. Die weltweite Wahrnehmung und die positiv besetzten Assoziationfelder, die durch die Symbole der Olympischen Spiele erzeugt werden, lassen die Olympischen Spiele trotz zunehmender Kritik in einigen westlichen Ländern immer noch zu einer ökonomisch wertvollen und interessanten Werbeplattform werden. Die Unternehmen, die Sponsoren oder Ausrüster scheinen ungemein von den Spielen zu profitieren, ansonsten würden sie nicht so viel Geld in ihre Sponsorships und Lizenzverträge investieren.

Das IOC besitzt alle Rechte an den Olympischen Emblemen. Es gibt für die NOCs, TV-Rechteinhaber und Sponsoren eigene Vermarktungssignets zur freien Nutzung, die die Ringe (Olympisches Symbol) enthalten (IOC 2020 a, $\$ 7-14$ Bye law 2.2). Die NOCs können diese dann auf ihrem Territorium vermarkten. Auf nationaler Ebene haben ferner die OCOGs das ausschließliche Recht an ihren spezifischen Emblemen und Maskottchen, da sie durch ein Joint-Marketing Agreement für vier Jahre allein über die Vermarktung im Ausrichterland bestimmen können, vor allem um die Exklusivität der Sponsoren zu schützen. In diesen vier Jahren wird das NOC des Ausrichterlandes aber angemessen vom OCOG an seinen Vermarktungseinnahmen beteiligt.

Die mediale Übertragung der Spiele ist bisher der Treiber des kommerziellen Erfolgs und stellt den größten Teil der Einnahmen dar. Die Rechteinhaber profitieren von den Spielen, indem sie durch diese einen Reputati- 
onsgewinn erzielen, die Rechte weitervermarkten oder auch durch hohe Einschaltquoten ihre Werbeflächen teurer weiterverkaufen können. In Bieterwettbewerben, die heute meist einer einseitigen, verdeckten Auktion gleichkommen, versuchen die verschiedenen Sendeanstalten, Medienagenturen, Netzbetreiber u.a. die Übertragungsrechte an attraktiven Sportveranstaltungen zu erwerben. Wie viel die Olympischen Spiele den Käufern wert sind, zeigt die Entwicklung des Verkaufs in Abb. 8. Interessant ist in diesem Zusammenhang die Digitalisierungsstrategie des IOC, siehe Beitrag von Kühner in diesem Sammelband). Demnach wird das IOC mit einem Olympic Broadcast Services (OBS) die Produktion der Fernsehbilder komplett übernehmen und allen rechtezahlenden Unternehmen und Sendern weltweit über eine OBS-Cloud zur Verfügung stellen. Das ermöglicht u.a. alle Medieninhalte auch nicht-linear zu sehen und ist damit auf die Generationen Y und Z, die einen starken digitalen Medienkonsum aufweisen, zugeschnitten. Die Vision, 365 Tage Olympism in Action über teilweise eigene Kanäle (Olympic Chanel) zu zeigen und die Partner über digitale Plattformen mit olympischen Inhalte zu versorgen, hatte IOC-Präsident Bach bereits 1994. Die absolute Kontrolle über alle Medieninhalte, bereitgestellt über eine gigantische Cloud und weltweit genutzten Servern ermöglicht es, jedem Konsumenten überall auf der Welt, zu jeder Zeit interessante olympische Inhalte zu zeigen (people centric).

Es ist festzustellen, dass der Medienmarkt in Nordamerika in den letzten 20 Jahren über $50 \%$ der IOC-Einnahmen erzeugte, wobei dieser Anteil von $61 \%$ (1997-2000) auf $51 \%$ (2013-2016) gefallen ist. In diesem Zeitraum blieb er in Europa konstant bei $23 \%$, während er in Asien im gleichen Zeitraum von $11 \%$ auf $16 \%$ gestiegen ist. Spätestens mit dem Kauf fast aller europäischen Rechte über den amerikanischen Kabelsender Discovery, der in Deutschland über seinen Sender Eurosport ausstrahlt, wurde eine neue Ära des Medienkonsums und der Medienrechteverkäufe eingeleitet. Die beiden amerikanischen Medienanbieter Discovery und NBC (Oympische Rechte bis 2032) sind zusammen aktuell für über $70 \%$ der Medienrechteeinnahmen des IOC verantwortlich. Dies zeigt die vorherrschende Dominanz der USA im Bereich der Medienrechte. 
Legacy - das Erbe der Olympischen Spiele

\begin{abstract}
Legacy
Legacy (engl.) bedeutet auch Vermächtnis oder Erbe und beinhaltet alle geplanten und ungeplanten, positiven und negativen, materiellen und immateriellen Veränderungen, die durch die Olympischen Spiele herbeigeführt werden und länger als die Spiele bestehen bleiben. Diese können die städtische Infrastruktur, Richtlinien und Gesetze, menschliches Können, gesellschaftliche Veränderungen, Erfindungen oder auch Auswirkungen auf die Umwelt umfassen. Alle diese sechs Bereiche lassen sich auf den Sport, die Wirtschaft, die Bildung oder andere Bereiche anwenden und zeigen damit die Breite von Legacy. Sie kann positiver, aber auch negativer Ausprägung sein, da sie die langfristigen Änderungen und die daraus folgenden Konsequenzen beschreibt.
\end{abstract}

Die Vision ist der Ausgangspunkt für den Planungsprozess der Olympischen Spiele. Sie muss zum einen den Visionen der Bewerberstadt/-region und ihrer Bevölkerung aber auch denen der olympischen Bewegung entsprechen. Jede Maßnahme, die für die Olympischen Spiele initiiert wird, sollte mit der Vision abgestimmt sein. So wird sichergestellt, dass die Stadt nicht nur Maßnahmen ergreift, die für die Austragung der Spiele wichtig sind, sondern auch für die Zeit danach. Nur so kann das Erbe (Legacy) positiver Art sein. Da es jedoch immer wieder Interessenkonflikte gibt, kann ein positives Vermächtnis für einen Akteur durchaus ein negatives Vermächtnis für einen anderen hinterlassen. Dies muss während des Planungsprozesses berücksichtigt und verantwortungsvoll abgewogen werden. Die Vision muss von der Gastgeberstadt entworfen werden, um einen maximalen Nutzen für die lokalen Akteure und Betroffenen zu gewährleisten. Sie muss aber auch in Absprache mit dem IOC erfolgen, um die Durchführbarkeit der Spiele zu garantieren.

Sobald die Olympischen Spiele an eine Stadt vergeben sind, beginnen die Stadt, die Regierung, private Investoren und das OCOG damit, die Standorte strukturell zu verändern. Eine strukturelle Veränderung kann die Infrastruktur, aber auch immaterielle Strukturen (wie z.B. Bildung oder Vernetzung von Akteuren) umfassen. Die strukturellen Veränderungen, die für oder aufgrund der Spiele auftreten, können sechs verschiedenen und sich nicht überschneidenden Facetten des Vermächtnisses zugeordnet werden:

1. Stadtentwicklung: z.B. Straßenbau, öffentliche Verkehrsmittel, neue oder renovierte Gebäude und Hotels, Sportstätten, Flughafenbau, aber 
auch die durch Neubauten und Gentrifizierung verursachten Verdrängungen und Umsiedlungen etc.

2. Umweltveränderung: z.B. Verbesserungen durch Abwasserreinigung, erneuerbare Energien, Wassereffizienz, umweltfreundliche Gebäude und Veranstaltungsorte, Revitalisierung zerstörter Biotope, aber auch Verschlechterung durch Naturzerstörung etc.

3. Politik und Governance (verantwortungsvolle politische Führung): neue Gesetze und Richtlinien sowie bessere Governance in Verbänden und Unternehmen wie beispielweise weniger Menschenrechtsverletzungen, Pressefreiheit, Gesetzgebung zum Ambush Marketing (Marketing ohne Rechteinhaber zu sein) aber eben auch mehr Transparenz, Mitsprache etc.

4. Fähigkeiten, Wissen und Netzwerke: Fähigkeiten, die durch die Bewerbung und die Ausrichtung der Spiele erworben wurden, geschaffenes oder geteiltes Wissen (z.B. wie man eine Sportveranstaltung organisiert), neue Netzwerke von Akteuren, die durch die Teilnahme an der Veranstaltung entwickelt wurden, was ggf. auch illegale Netzwerke einschließen könnte.

5. Geistiges Eigentum: geistiges Eigentum, das zu Innovationen oder einem zusätzlichen oder verringerten Markenwert führt, Erfindungen, die für oder wegen der Olympischen Spiele gemacht wurden wie zum Beispiel Musikstücke, Piktogramme, Sicherheitskonzepte, Verkehrsleitsysteme und Technologien.

6. Überzeugungen und Verhalten: neue oder veränderte Überzeugungen, z.B. in Bezug auf das Image eines Landes (sowohl positiv als auch negativ), Verhaltensänderungen, z.B. in Bezug auf die Servicequalität, die Nutzung öffentlicher Verkehrsmittel.

Viele dieser Legacies verändern die Qualität eines Standorts und haben damit letztlich wirtschaftliche Auswirkungen. Beispielweise wird eine Stadt somit für den Tourismus attraktiver, wird als Industriestandort gewählt oder bekommt eine neue Universität. Diese Wirkung ist sehr schwer zu messen, soll dem Leser aber ein Gefühl vermitteln, dass die Investitionen in die Olympischen und Paralympischen Spiele nicht nur für die Tage der eigentlichen Veranstaltungen vorhalten. Daneben sind diese langfristigen Auswirkungen schwer zu kommunizieren. Dies nämlich ist einer der wesentlichen Gründe, warum Bürgerreferenden in Deutschland und anderen westlichen Nationen in den vergangenen Jahren oft verloren gingen, z.B. München 2022, Hamburg 2024. 
Der Zeitpunkt des Hamburger Referendums für/gegen Olympia 2024 wurde von den Befürwortern der Spiele im Nachhinein kritisiert. Sie argumentierten, dass deren Finanzierung zu dem Zeitpunkt noch nicht geklärt war und dies für viele Bürger*innen ein bekannter Ablehnungsgrund war (Nielsen, 2015). Ein Volksentscheid sollte erst dann durchgeführt werden, wenn die Kosten der Spiele auch wirklich richtig bestimmt werden können. Dies ist jedoch sieben Jahre vor den Spielen nach Preuss et al. (2019) noch nicht möglich. Es ist weithin bekannt, dass der Befragungszeitraum, -zeitpunkt und Minderheitenmobilisierung das Ergebnis eines Volksentscheides maßgeblich beeinflussen. Mehrere Autor*innen zeigten, dass sich direktdemokratische Verfahren eher hinderliche als nützlich für eine Ausweitung von Staatsausgaben erwiesen (Freitag, Vatter \& Müller, 2003) und einen „Zügelungseffekt auf das Ausgabenniveau der Staatstätigkeit“ (Wagschal und Obinger, 2000, S. 493) haben. Moser und Obinger (2007) konnten belegen, dass es bei Volksentscheiden eher zu einer Bestätigung des Status Quo kommt, als dass Staatsausgaben befürwortet werden. Diese Studienergebnisse offenbaren die Schwierigkeit der Legitimierung von Olympischen Spielen in Deutschland über einen Volksentscheid, sofern hohe Infrastrukturinvestitionen über Steuermittel getätigt werden müssen.

Preuss et al. (2019) konnten nachweisen, dass die Organisation der Spiele zu Kostenüberschreitungen führt, wobei die Einnahmeüberschreitungen diese jedoch regelmäßig vollständig kompensieren (Abb. 9). Eine Finanzierung der Spiele erfolgt obendrein fast ausschließlich über private Mittel (IOC Beitrag, Sponsoren, Eintrittskarten). Bei guter Aufklärung der Bevölkerung dürfte es hier wegen dieser Erkenntnisse keinen Grund für eine Ablehnung zukünftiger Spiele wegen der Organisationskosten geben. 
Abbildung 9: Einnahme und Kostenüberschreitung von Organisationskomitees

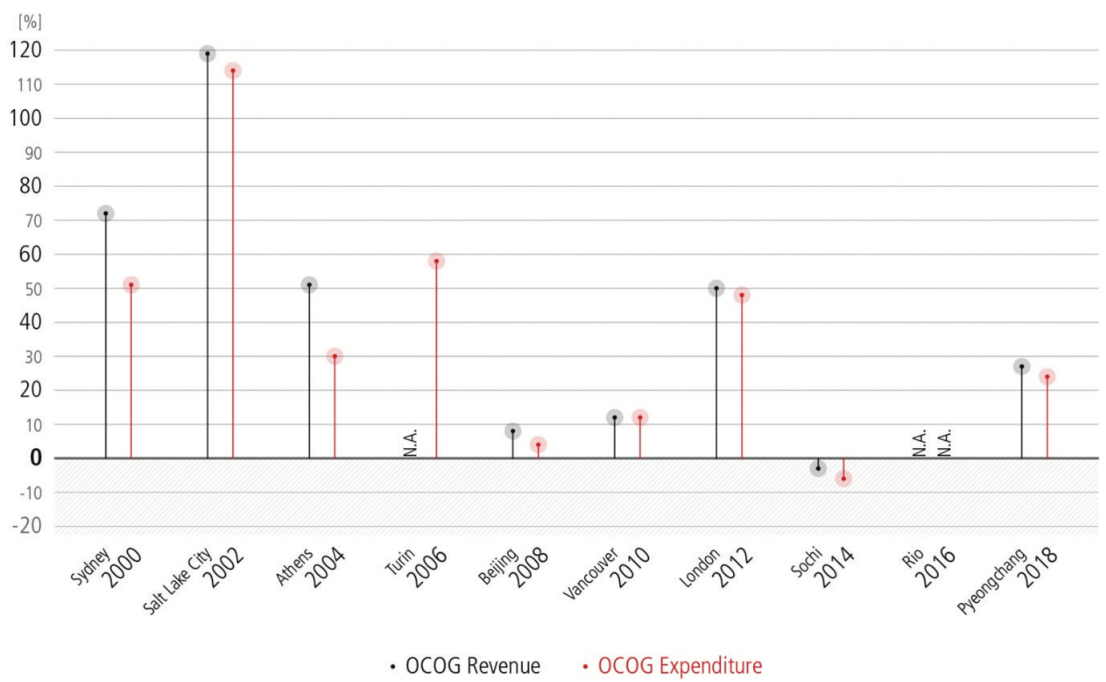

(Preuss et al., 2019, S. 115)

Die Abb. 9 zeigt allerdings nicht die zu erwartenden Kostenüberschreitungen bei Investitionen in die Infrastruktur. Sofern aber keine Infrastruktur geplant wird, die allein den Olympischen Spielen dienen und die keinen Folgenutzen haben, sollten Infrastrukturveränderungen höchstens anteilig als Kosten Olympischer Spiele zählen. Es sollten keine Stadien, Autobahnen und Flughäfen in Deutschland allein wegen der Olympischen und Paralympischen Spiele gebaut werden, sondern immer einer Vision folgen, die auch nach den Spielen nachhaltigen Nutzen für die Bevölkerung stiftet (z.B. Berliner Olympiastadion, Münchner Olympiapark).

In Deutschland steigt die Bedeutung von regionalen direktdemokratischen Verfahren, sodass künftige Bewerbungen um Olympische Spiele vermutlich wieder an ein positives Referendum gekoppelt sein werden. Daher stellt sich die Frage, wie sich die Chancen auf den positiven Ausgang eines solchen Referendums erhöhen lassen.

\section{Diskussion zur ökonomischen Effizienz des bestehenden Systems}

Der bisherige langsame Wandel des IOC und eine damit teilweise einhergehende Ineffizienz lässt sich durch spezifische Bedingungen dieser Organisation zurückführen, die Anastasiadis \& Spence (2019) als organisa- 
tionale Pathologie beschreiben. Diese führte in den Augen der Öffentlichkeit zu Einbußen an Legitimität. Ohne moralische Legitimität wird das IOC in der Öffentlichkeit und Sportpolitik aber immer wieder auf aktiven oder passiven Widerstand stoßen. Zusammenarbeit funktioniert dann nur noch durch finanzielle Gegenleistungen, da Vertrauen weniger vorhanden ist (Finnemore 2009). Diesen Verlust an moralischer Legitimität versucht das IOC heute sehr aktiv und auch effektiv mit Hilfe der Agenda 2020 (IOC 2013) und aktuelle mit der Agenda 2020+5 (IOC, 2021) zu beseitigen.

\section{Agenda 2020 \& Agenda 2020+5}

Die Olympische Agenda 2020+5 baut auf den Ergebnissen der Olympischen Agenda 2020 (2014 vom IOC verabschiedet) auf. Die Agenda 2020 hat die Olympische Bewegung durch die Einführung von 40 Änderungen gestärkt (z. B. die Olympischen Spiele zukunftsfähig gemacht, die Olympischen Werte gesichert und die Rolle des Sports in der Gesellschaft gefestigt). Diese Errungenschaften haben ein solides Fundament für die Zukunft gelegt.

Die 15 Empfehlungen der Agenda 2020+5, die von der IOC-Session 2021 ins Leben gerufen wurden, entstanden in einem inklusiven und gemeinschaftlichen Prozess aus Vorschlägen rund um die Welt. Die neuen Empfehlungen basieren auf vom IOC identifizierten „Schlüsseltrends“, die höchstwahrscheinlich in der Post-Corona-Ära an Bedeutung gewinnen werden und bei denen der Sport und die olympischen Werte eine Schlüsselrolle spielen könnten. Es sind Solidarität, Digitalisierung, Nachhaltigkeit, Glaubwürdigkeit und finanzielle Absicherung.

Allmählich wird die Selbstreferenz zurückgefahren, indem es zu einer Öffnung der IOC-Kommissionen für Experten und Stakeholder kommt und einer Öffnung von Veranstaltungen (IOC-Session, IOC Forum etc.) für die Medien und Olympiagegner. Ein externes Rechnungsprüfsystem mit höchstem Standard und von PricewaterhouseCoopers geleitet wurde eingeführt. Die Ethikkommission ist mehrheitlich durch Externe besetzt und die negative Wahrnehmung des IOC durch die Bevölkerung, die sich deutlich in Referenden gegen die Ausrichtung Olympischer Spiele zeigte (Preuss, Scheu \& Weitzmann 2020), kann allmählich durch zahlreiche Veränderungen der Ausrichtungsvorgaben (IOC New Norm) und durch die Veröffentlichung des Host City Contracts (erhöhte Transparenz) bearbeitet und positiv stimuliert werden. Der Aufbau moralischer Legitimität dauert aber lange. Dennoch sollte dies nicht davon ablenken, dass sich Deutschland wieder um die Olympischen und Paralympischen Spiele 
bewerben sollte, denn aus ökonomischer Sicht dürfte die Grundidee dieser Spiele und des IOC mit seinem pro-sozialen Bestreben, Bildung und Frieden besser zum heutigen Zeitgeist passen.

\section{Literatur:}

Anastasiadis, S., Spence, L. J. (2019). An Olympic-sized Challenge: Effect of Organizational Pathology on Maintaining and Repairing Organizational Legitimacy in Sports Governing Bodies. British Journal of Management, 31, 24-41.

Barnett, M., Finnemore M. (1999). The politics, power, and pathologies of international organizations', International Organization, 53, 699-732.

Finnemore, M. (2009). Legitimacy, hypocrisy, and the social structure of unipolarity, World Politics, 61, 58-85.

Freitag, M., Vatter, A., Müller, Ch. (2003). Bremse oder Gaspedal? Eine empirische Untersuchung zur Wirkung der direkten Demokratie auf den Steuerstaat. Politische Vierteljahresschrift, 44, 348-369

Geeraert, A., Mrkonjic, M., Chappelet, J.-L. (2014). A rationalist perspective on the autonomy of international sport governing bodies: towards a pragmatic autonomy in the steering of sports, International Journal of Sport Policy and Politics, 7, 473-488.

Green, J. F. (2014). Rethinking Private Authority: Agents and Entrepreneurs in Global Environmental Governance. Princeton, NJ: Princeton University Press.

IOC (2013). Olympic Agenda 2020: 20+20 Recommendations. Lausanne: International Olympic Committee.

IOC (2016). Marketing Report Rio 2016. Lausanne: International Olympic Committee.

IOC (2017). Host City Contract - Principles Games of the XXXIII Olympiad in 2024. Lausanne: International Olympic Committee.

IOC (2018). Marketing Report PyeongChang 2018. Lausanne: International Olympic Committee.

IOC (2019). The IOC Annual Report 2019. Credibility Sustainability Youth. Lausanne: International Olympic Committee.

IOC (2020a). Olympic Charter, in Force as from 17 July 2020. Lausanne: International Olympic Committee.

IOC (2020b). Recognised Organisations. Abgerufen am 23.11.2020 unter https:// www.olympic.org/ioc-governance-affiliate-organisations

IOC (2020c). Ethics 2020. Lausanne: International Olympic Committee.

IOC (2020d). IOC increases Olympic Solidarity fund by $16 \%$ overall and by $25 \%$ for direct atblete support programmes. Abgerufen am 30.11.2020 unter https://www. olympic.org/news/ioc-increases-olympic-solidarity-fund-by-16-per-cent-overalland-by-25-per-cent-for-direct-athlete-support-programmes 
IOC (2020e). IOC Good Governance. Abgerufen am 18.12.2020 unter https://www. olympic.org/good-governance

IOC (2020f). IOC Marketing Fact File. Abgerufen am 7.1.2021 unter https://stillme dab.olympic.org/media/Document\%20Library/OlympicOrg/Documents/IOCMarketing-and-Broadcasting-General-Files/Olympic-Marketing-Fact-File.pdf\#_ $\mathrm{ga}=2.109772933 .1643799770 .1610010563-443683503.1588420897$

IOC (2021). Olympic Agenda 2020+5. Lausanne: International Olympic Committee.

Kobrin, S. J. (2009). Private political authority and public responsibility: transnational politics, transnational firms, and human rights, Business Ethics Quarterly, 19, 349-374.

MacAloon, J. J. (2011). Scandal and governance: inside and outside the IOC 2000 Commission, Sport in Society, 14, 292-308.

Moser, J., \& Obinger, H. (2007). Schlaraffenland auf Erden? Auswirkungen von Volksentscheiden auf die Sozialpolitik. In M. Freitag \& U. Wagschal (Hrsg.), Direkte Demokratie. Bestandsaufnahmen und Wirkungen im internationalen Vergleich, (S. 331-362). Berlin: LIT Verlag.

Nielsen (2015). Bevölkerungsrepräsentative Befragung zur Akzeptanz der Bewerbung Hamburgs für die Ausrichtung der Olympischen und Paralympischen Spiele 2024. Berichtsband Fieberkurven, Hamburg Olympiabewerbung, 15. November 2015. Typoskript.

Owen, D. (2019). Exclusive: IOC distributions to United States Olympic and Paralympic Committee jump on back of surging sponsorship revenue. Abgerufen am 8.1.2021 unter https:/www.insidethegames.biz/articles/1081565/ioc-distributions-tousopc-jump

Preuss, H., Andreff, W., \& Weitzmann, M. (2019). Cost and Revenue Overruns of the Olympic Games 2000-2018. Wiesbaden: Springer Fachmedien Wiesbaden.

Preuss, H., Scheu, A. \& Weitzmann, M. (2020). Referendums at Olympic Games. In Chatziefstathiou, D., Garcia, B. \& Séguin, B. (Eds). Handbook on the Olympic and Paralympic Games. Routledge.

United Nations (2014). UN, Olympic Committee sign formal agreement on role of sport in development, peace, Press release, 28 April. Abgerufen am 23.11.2020 unter http://www.un.org/apps/news/story.asp?NewsID=47671

WADA (2021). Contributions to WADA's budget 2021. Abgerufen am 24.11.2020 unter https://www.wada-ama.org/sites/default/files/resources/files/wada_contrib utions_2021_update_en.pdf

Wagschal, U., \& Obinger, H. (2000). Der Einfluss der Direktdemokratie auf die Sozialpolitik. Politische Vierteljahresschrift, 41 (3), 466-497. 
